\title{
Long term clinical impact of successful recanalization of chronic total occlusion in patients with and without type 2 diabetes mellitus
}

\author{
Chuan-Tsai Tsai ${ }^{1,3}$, Wei-Chieh Huang ${ }^{1,2,3}$, Hsin-I Teng ${ }^{1,2,3}$, Yi-Lin Tsai ${ }^{1,2,3}$ and Tse-Min Lu 1,2,3*
}

\begin{abstract}
Background: Diabetes mellitus is one of the risk factors for coronary artery disease and frequently associated with multivessels disease and poor clinical outcomes. Long term outcome of successful revascularization of chronic total occlusions (CTO) in diabetes patients remains controversial.

Methods and results: From January 2005 to December 2015, 739 patients who underwent revascularization for CTO in Taipei Veterans General Hospital were included in this study, of which 313 (42\%) patients were diabetes patients. Overall successful rate of revascularization was 619 (84\%) patients whereas that in diabetics and non-diabetics were 265 (84\%) and 354 (83\%) respectively. Median follow up was 1095 days (median: 5 years, interquartile range: 1-10 years). During 3 years follow-up period, 59 (10\%) in successful group and 18 (15\%) patients in failure group died. Although successful revascularization of CTO was non-significantly associated with better outcome in total cohort (hazard ratio (HR): 0.593, 95\% confidence interval (Cl) 0.349-0.008, P: 0.054), it might be associated with lower risk of all-cause mortality (HR: 0.307, 95\% Cl 0.156-0.604, P: 0.001) and CV mortality (HR: 0.266, 95\% Cl 0.095-0.748, P: 0.012) in diabetics (P: 0.512). In contrast, successful CTO revascularization didn't improve outcomes in non-diabetics (all $P>0.05$ ). In multivariate cox regression analysis, successful CTO revascularization remained an independent predictor for 3-years survival in diabetic subgroup (HR: 0.289, 95\% Cl 0.125-0.667, P: 0.004). The multivariate analysis result was similar after propensity score matching (all-cause mortality, HR: 0.348, 95\% Cl 0.142-0.851, P: 0.021).
\end{abstract}

Conclusions: Successful CTO revascularization in diabetes may be related to better long term survival benefit but not in non-diabetic population.

Keywords: Chronic total occlusion, Percutaneous coronary intervention, Diabetes mellitus

\section{Background}

Approximately $15 \%$ to $30 \%$ of patients who received coronary angiography had one or multiple chronic total occlusion (CTO) of coronary arteries [1, 2]. Percutaneous coronary intervention (PCI) for CTO is technical

\footnotetext{
*Correspondence: tmlu@kimo.com

${ }^{1}$ Division of Cardiology, Department of Medicine, Taipei Veterans General Hospital, No. 201, Sec. 2, Shih-Pai Road, Taipei, Taiwan

Full list of author information is available at the end of the article
}

challenging and always need ample experiences, dedicated techniques and advanced interventional devices. Moreover, PCI for CTO lesion is related to higher radiation exposure to the patient and operator, more contrast volumes and increased risk for peri-procedural complications. Successful PCI of CTO lesion has been reported to be associated with improved left ventricular function and better clinical outcomes; while other studies showed negative results [3, 4]. On the other hand, Diabetes mellitus, a well-established risk factor of atherosclerosis, is always 
associated with more complex atherosclerotic coronary artery disease, including multi-vessel disease, diffuse and small vessel disease, and heavily calcified lesions. In addition, diabetes has been reported to be associated with longer length of CTO lesions $[5,6]$, and the treatment for CTO lesions in diabetes are more complex with lower success rate [7]. Even after successful revascularization, there were higher in-hospital major adverse cardiovascular and cerebrovascular events (MACCE) in diabetic patients comparing to non-diabetic patients [8]. Besides, diabetes is related to higher incidence of mortality and revascularization after CTO PCI up to 5 years compared to non-diabetics [9]. However, long term impact of successful revascularization for CTO lesions in diabetes population remains unknown and controversial. Therefore, in this study we aimed to investigate the long-term clinical outcomes in diabetic patients undergoing CTO PCI.

\section{Methods}

\section{Study population}

We enrolled patients who underwent CTO PCI at Taipei Veterans General Hospital. From January 2005 to December 2015, a total of 739 patients underwent intervention for CTO lesions. CTO lesions were defined as complete blockage of a coronary artery for longer than 3 months with thrombolysis in myocardial infarction (TIMI) 0 . All patients had at least 1 CTO lesion and met the indication of recanalization of CTO lesion. Indications for CTO revascularization were as follows: (1) angina resistant to pharmacological therapy, (2) exercise-induced symptoms or (3) exercise induced evidence of myocardial ischemia. Patients with acute coronary syndrome and end stage renal disease on renal replacement therapy were excluded. Diabetes mellitus (DM) was defined as a fasting plasma glucose of at least $126 \mathrm{mg} / \mathrm{dl}$, or $2 \mathrm{~h}$ postprandial plasma glucose of $200 \mathrm{mg} / \mathrm{dl}$ or glycated hemoglobin of at least $6.5 \%$ or random plasma glucose of at least $200 \mathrm{mg} /$ $\mathrm{dl}$ in presence of classic symptoms of hyperglycemia [10]. Left ventricular ejection fraction was measured from transthoracic echocardiography or left ventriculography. Renal function was classified according to estimated glomerular filtration rate (eGFR) calculated by the modified diet in renal disease equation for Chinese (MDRDc) [11]. Patient's demographics, coronary angiography, PCI records, in-hospital treatments, and in-hospital laboratory tests were extracted from web based electronic medical system of our hospital.

\section{Coronary angiography and percutaneous coronary intervention $(\mathrm{PCI})$ procedure}

Diagnostic coronary angiography was evaluated carefully by experience cardiologists for the morphology of
CTO lesion and collaterals. J-CTO score was calculated as previously reported [12]. Radial or femoral artery approaches/uni- or bi-directional approaches were used for diagnostic angiography and percutaneous coronary intervention according to standardized protocol of cardiac catheterization laboratory. After unfractionated heparin (10,000 IU bolus) was administered before the procedure to achieve an activated clotting time $>300 \mathrm{~s}$, we routinely tried antegrade approach first. Antegrade approach includes single wire technique with wire escalation and parallel wire technique. If antegrade approach did not work, we would try retrograde approach if there are suitable collaterals available. After wire crossing collateral retrogradely, we always tried retrograde wiring technique, kissing wire technique, and reverse controlled antegrade and retrograde subintimal tracking (CART) technique. However, if both approaches failed and the CTO lesion morphology is suitable, intravascular ultrasound (IVUS) guided wiring re-entry technique would be tried. The PCI procedure was considered angiographically successful if residual stenosis $<30 \%$ and coronary Thrombolysis in Myocardial Infarction grade 3 flow were obtained at the end of the procedure.

Dual antiplatelets therapy was started on the day before PCI procedure or immediately after the procedure, and all patients received aspirin $(100 \mathrm{mg} /$ day $)$ indefinitely and clopidogrel (300 to $600 \mathrm{mg}$ loading dose, and $75 \mathrm{mg}$ maintenance per day) for at least 3 month (bare metal stent (BMS)) or 12 months (drug-eluting stent, DES). After 1 year, aspirin or clopidogrel was maintained lifelong. Medications for treatment of angina pectoris (calcium channel blockers, beta-blockers and nitrates) were continued.

\section{Clinical outcomes}

Clinical endpoints were 3 years all-cause mortality, cardiovascular $(\mathrm{CV})$ mortality, nonfatal myocardial infarct (MI) and composite endpoints (MACE). MACE was the composite endpoint of all-cause mortality, CV mortality and nonfatal MI. Myocardial infarction was defined as the presence of significant new $\mathrm{Q}$ waves in at least 2 electrocardiographic leads or of symptoms compatible with $\mathrm{MI}$ associated with increase in creatine kinase-MB fraction $\geq 3$ times the upper limit of the reference range. Periprocedural cardiac enzymes elevation was excluded from this definition of MI. Cardiovascular death was diagnosed as any death with definite cardiovascular cause or any death that was not clearly attributed to a non-cardiovascular cause. Hospital re-admission and outpatient clinic records from our hospital web-based system were obtained for clinical outcomes. In addition, patients were contacted by research coordinator by telephone interview at the end of study period if loss of follow up in our 
hospital. The study protocol was approved by the Institutional Review Board at Taipei-Veterans General Hospital, and all participants provided written informed consent.

\section{Statistical analysis}

Continuous variables were compared with Student's t-test and were expressed as mean \pm standard deviation (SD). Categorical data were tested using Chi-square test and presented as frequencies and percentages. Propensity score matching was performed using logistic regression model. We adjusted variables that were known as confounding factors (age, sex, renal function). Success and failure to revascularize groups were matched by a 4:1 matching protocol according to propensity scores with the width equal to 0.05 of the standard deviation. Kaplan-Meier estimates were used for survival curves which were compared with log-rank test. Multivariate analyses were performed with a cox proportional hazards model and confounders were selected according to statistically significance $(\mathrm{P}<0.05)$ in univariate analysis which were age, renal function, prior stroke, peripheral arterial disease, renal function and left ventricular ejection fraction (LVEF). P-value of less than 0.05 was considered as statistically significance. All statistically analyses were performed with the use of SPSS 17.0 software (SPSS Inc, Chicago, IL, USA).

\section{Results}

From January 2005 to December 2015, a total of 973 patients were found to have CTO lesion during coronary angiography in which 739 patients received revascularization at Taipei Veterans General Hospital (Fig. 1). The mean age was $68 \pm 13$ years, and most of the patients were male $(675,91 \%)$. Most patients had multivessel disease $(607,82 \%)$, and right coronary artery was the most common treated CTO vessel (362, 49\%). 76 (10\%) patients underwent bypass surgery prior to CTO PCI. The mean J-CTO score was $2.5 \pm 0.97$. Chronic kidney disease was present in 165 patients (22\%). Most CTO lesions were crossed by antegrade wire escalation technique/parallel wire technique, while the retrograde approach was tried in only 56 patients (7.6\%). Successful revascularization was achieved in 619 (84\%) patients of entire cohort. Compared to patients with successful revascularization, the failed PCI group had poorer renal function, higher prevalence of multivessel CTO, and longer CTO length. Drug eluting stent was used in 317 (43\%) patients. The baseline clinical and angiographic characteristics are shown in Table 1.

The incidences of clinical outcomes (all-cause mortality, CV mortality, nonfatal MI and MACE) followed up for 3 years (median: 5 years, interquartile range: 1-10 years) were summarized in Table 2 (Fig. 2a). In entire population, there were no significant differences in the incidence of all-cause mortality, CV mortality, nonfatal MI and MACE (hazard ratio (HR): 0.593, 95\% confidence interval (CI) 0.349-1.008, P: 0.054; HR: 0.472, 95\% CI 0.217-1.024, P: 0.057; HR: 0.867, 95\% CI 0.294-2.563, P: 0.797; HR: 0.734, 95\% CI 0.449-1.200, P: 0.218 respectively) between successful revascularization group and failed revascularization group. Three years all-cause mortality was statistically significant

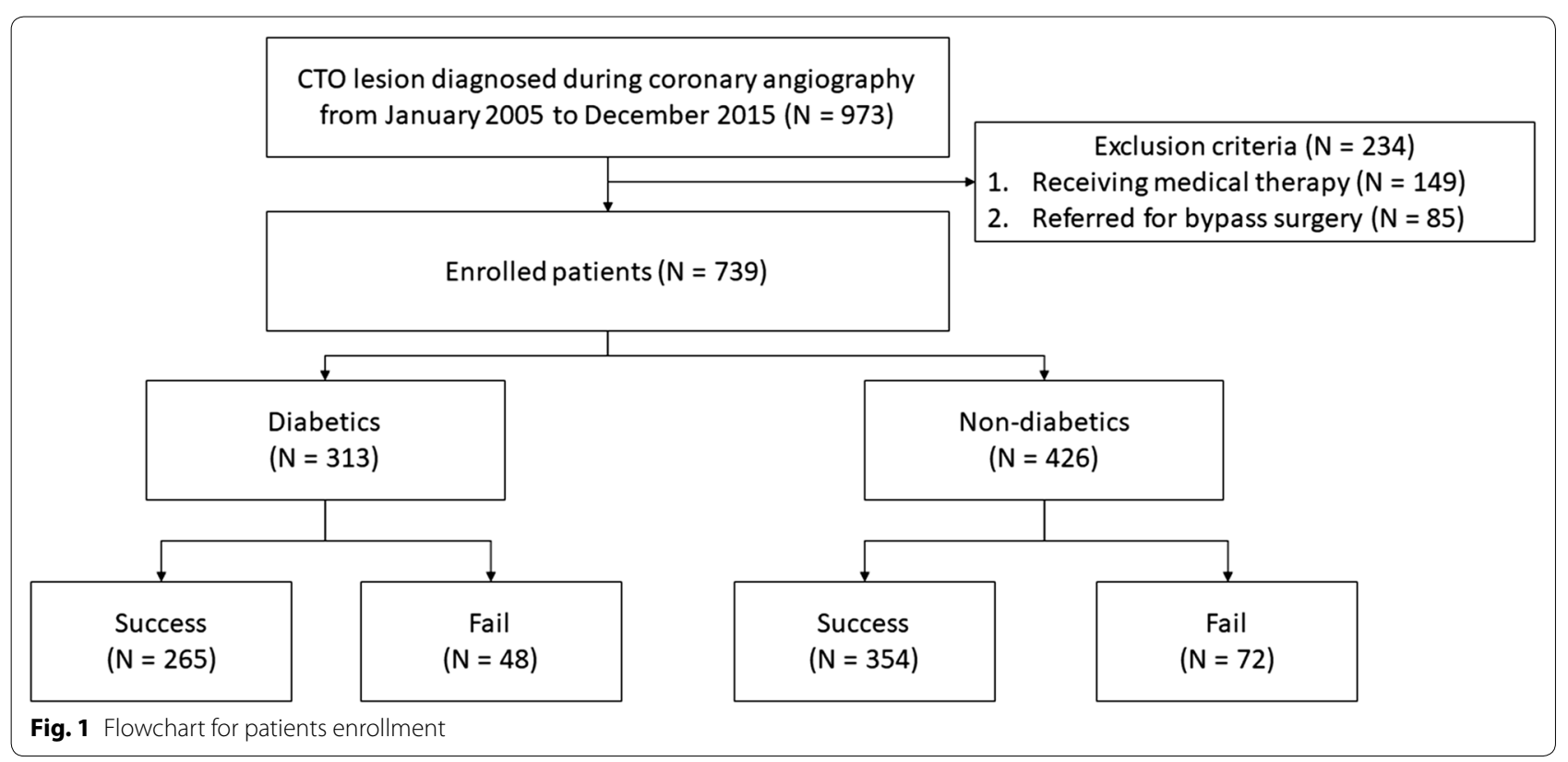


Table 1 Baseline demographics and angiographic characteristics of entire population with successful and Failed revascularization

\begin{tabular}{|c|c|c|c|}
\hline & \multicolumn{3}{|c|}{ Entire population $(n=739)$} \\
\hline & Success $(n=619)$ & Failed $(n=120)$ & $P$ value \\
\hline Age (years) & $67 \pm 13$ & $69 \pm 13$ & 0.14 \\
\hline Gender (male) & $568(92)$ & $107(89)$ & 0.38 \\
\hline Hypertension & $467(75)$ & $96(80)$ & 0.35 \\
\hline $\mathrm{DM}$ & $265(43)$ & $48(40)$ & 0.61 \\
\hline Hyperlipidemia & $267(43)$ & $53(44)$ & 0.84 \\
\hline Prior stroke & $51(8)$ & $11(9)$ & 0.72 \\
\hline $\mathrm{PAD}$ & $34(6)$ & $11(9)$ & 0.14 \\
\hline Smoking & $253(41)$ & $47(39)$ & 0.76 \\
\hline Prior Ml & $135(22)$ & $24(20)$ & 0.72 \\
\hline Prior PCl & $317(51)$ & $66(55)$ & 0.49 \\
\hline Prior CABG & $61(10)$ & $15(13)$ & 0.41 \\
\hline LVEF (\%) & $49 \pm 12$ & $48 \pm 13$ & 0.61 \\
\hline eGFR, MDRDc (ml/min) & $84 \pm 33$ & $76 \pm 34$ & 0.01 \\
\hline $\mathrm{LDL}(\mathrm{mg} / \mathrm{dl})$ & $102 \pm 35$ & $97 \pm 32$ & 0.19 \\
\hline $\mathrm{HbA1C}(\%)$ & $8.0 \pm 3.8$ & $7.6 \pm 1.7$ & 0.51 \\
\hline \multicolumn{4}{|l|}{ Location of CTO } \\
\hline LAD & $271(44)$ & $46(38)$ & 0.31 \\
\hline LCX & $159(26)$ & $37(31)$ & 0.26 \\
\hline RCA & $293(47)$ & $63(53)$ & 0.32 \\
\hline J CTO score & $2.45 \pm 1.00$ & $2.55 \pm 0.96$ & 0.32 \\
\hline MVD & $501(81)$ & $106(88)$ & 0.07 \\
\hline Lesion length (mm) & $38 \pm 18$ & $32 \pm 11$ & 0.01 \\
\hline Lesion width (mm) & $3.2 \pm 3.6$ & $3.0 \pm 0.5$ & 0.73 \\
\hline Primary retrograde & $48(8)$ & $8(7)$ & 0.34 \\
\hline
\end{tabular}

Values are given as mean and standard deviation or numbers and percentages $D M$ diabetes mellitus, $P C l$ percutaneous coronary intervention, $P A D$ peripheral arterial disease, $M I$ myocardial infarct, $P C l$ percutaneous coronary intervention, $C A B G$ coronary artery bypass graft surgery, $L V E F$ left ventricular ejection fraction eGFR estimated glomerular filtration rate, MDRDc modification of diet in renal disease Chinese, $L D L$ low density lipoprotein, $C T O$ chronic total occlusion, $C A D$ coronary artery disease, $L A D$ left anterior descending, $L C x$ left circumflex, $R C A$ right coronary artery, MVD multivessel significant coronary artery disease

higher in diabetes population compared to non-diabetes (P: 0.03) (Fig. 2b). Subgroup analysis showed that successful revascularization in diabetic population exhibited better survival benefit compared to that of non-diabetics (HR: 0.306, 95\% CI 0.156-0.601 vs HR: $1.330,95 \%$ CI $0.519-3.407$, interaction P: 0.013) (Fig. 2c).

Totally 313 (42\%) patients are diabetics and 426 (58\%) patients are non-diabetics. Among diabetic patients, $68(21.7 \%)$ patients received insulin treatment. Compared to non-diabetic patients, diabetic patients were significantly older, with higher percentage of hypertension, worse renal function, multi-vessels disease, and reduced LVEF. There was no statistically difference in J-CTO score between two groups of diabetics and non-diabetics. Syntax score was statistically significant higher in failed group of diabetics but not different in non-diabetics. However, the revascularization successful rate was similar between diabetic patients (265, $84 \%)$, and non-diabetics patients $(354,83 \%, \mathrm{P}=0.614)$. The angiographic procedure and characteristics were not significantly different between the two groups (Table 3).

Risk of long-term all-cause mortality, CV mortality and MACE in successful recanalization group were significantly lower comparing to those of failed group in diabetics subgroup (HR: 0.307, 95\% CI 0.156-0.604, P: 0.001; HR: 0.266, 95\% CI 0.095-0.748, P: 0.013; HR: 0.454, 95\% CI $0.246-0.837$, P: 0.011 respectively), whereas there were no significant differences in these endpoints in non-diabetes population (all-cause mortality: HR: 1.334, 95\% CI 0.521-3.417, P: 0.548; CV mortality: HR: 0.885, 95\% CI 0.252-3.107, P: 0.849; nonfatal MI: HR: 1.423, 95\% CI 0.175-11.565, P: 0.741; and MACE: HR: 1.351, 95\% CI 0.573-3.188, P: 0.491) (Table 2). Figure 3 shows the cumulative survival curves free from 3-year all-cause mortality determined using the Kaplan-Meier method between successful and failed revascularization group in entire population and diabetic/non-diabetic patients, with the outcome significantly worse only in those diabetic patients undergoing failed revascularization procedure ( $\mathrm{P}:$ 0.001). Periprocedural complications were summarized in Table 4 . There was no statistically significant difference in complication rate between two groups of diabetes. In non-diabetics, there was higher prevalence of pericardial effusion which required pericardiocentesis in failure group ( $P: 0.04)$.

\section{Propensity score-adjusted clinical outcomes}

To reduce the effect of treatment selection bias and compensate for potential confounding factors in this observational study, we calculated the propensity score by using multiple logistic regression analysis incorporating patient's age, gender, renal function variables. After propensity score matching, there were no significant differences in the baseline characteristics between the successful PCI and failed PCI group of diabetes population (Table 5). In propensity score matched population, successful CTO revascularization was associated with reduced 3-years all-cause mortality and CV mortality only in diabetes population (all-cause mortality; $\mathrm{HR}$ 0.386, 95\% CI 0.188-0.789, P: 0.009, CV mortality; 0.280, 95\% CI 0.094-0.834, P: 0.018, Fig. 3c). In contrast, the risks of non-fatal MI and MACE were not reduced after successful CTO recanalization in diabetic patients (HR: 0.584, 95\% CI 0.154-2.210, P: 0.429; HR: 1.511, 95\% CI 0.338-6.753, P: 0.589, Table 2). In addition, there were 
Table 2 Various clinical outcomes up to 3 years by Kaplan-Meier curved analysis

\begin{tabular}{|c|c|c|c|c|}
\hline \multirow[t]{3}{*}{ Entire population } & \multicolumn{4}{|c|}{ Incidence of event at 3 years [n (\%)] } \\
\hline & \multicolumn{2}{|l|}{ Procedure } & \multirow[t]{2}{*}{$\mathrm{HR}(95 \% \mathrm{Cl})$} & \multirow[t]{2}{*}{ P value } \\
\hline & Successful PCI $(n=619)$ & Failed PCI $(n=120)$ & & \\
\hline All cause mortality & $59(10)$ & $18(15)$ & $0.593(0.349-1.008)$ & 0.054 \\
\hline CV mortality & $22(4)$ & $9(8)$ & $0.472(0.217-1.024)$ & 0.057 \\
\hline Nonfatal Ml & $18(3)$ & $4(3)$ & $0.867(0.294-2.563)$ & 0.797 \\
\hline MACE & $80(13)$ & $20(17)$ & $0.734(0.449-1.200)$ & 0.218 \\
\hline Diabetes patients & Successful PCI ( $n=265)$ & Failed PCI $(n=48)$ & & \\
\hline All cause mortality & $25(9)$ & $13(27)$ & $0.307(0.156-0.604)$ & 0.001 \\
\hline CV mortality & $9(3)$ & $6(13)$ & $0.266(0.095-0.748)$ & 0.012 \\
\hline Nonfatal Ml & $11(4)$ & $3(6)$ & $0.652(0.182-2.338)$ & 0.512 \\
\hline MACE & $39(15)$ & $14(29)$ & $0.454(0.246-0.837)$ & 0.011 \\
\hline Non diabetes patients & Successful PCI $(n=354)$ & Failed PCI $(n=72)$ & & \\
\hline All cause mortality & $34(10)$ & $4(7)$ & $1.334(0.521-3.417)$ & 0.548 \\
\hline CV mortality & $13(4)$ & $3(4)$ & $0.885(0.252-3.107)$ & 0.849 \\
\hline Nonfatal Ml & $7(2)$ & $1(1)$ & $1.423(0.175-11.565)$ & 0.741 \\
\hline MACE & $41(12)$ & $6(8)$ & $1.351(0.573-3.188)$ & 0.491 \\
\hline Diabetes patients after matching & Successful PCI $(n=188)$ & Failed PCI $(n=47)$ & & \\
\hline All cause mortality & $21(11)$ & $12(26)$ & $0.386(0.188-0.789)$ & 0.009 \\
\hline CV mortality & $7(4)$ & $6(13)$ & $0.268(0.090-0.798)$ & 0.018 \\
\hline Nonfatal Ml & $8(4)$ & $3(6)$ & $0.584(0.154-2.210)$ & 0.429 \\
\hline MACE & $28(15)$ & $13(28)$ & $1.511(0.338-6.753)$ & 0.589 \\
\hline
\end{tabular}

CV cardiovascular, MI myocardial infarct, MACE major adverse cardiovascular events (defined as the composite of all-cause mortality, cardiovascular mortality, and non fatal myocardial infarct)

no significant differences in clinical outcomes after successful or failed CTO recanalization in propensity score matched non-diabetic group.

In multivariate Cox-regression analysis, successful CTO revascularization remained an independent predictor of 3 years all-cause mortality in diabetic patients (HR: 0.289, 95\% CI 0.125-0.667, P: 0.004) after adjusting age, renal function, prior stroke, prior peripheral arterial disease, left ventricular ejection fraction (Table 6). The results of univariate analysis and multivariate analysis were similar after propensity score matching (Table 7).

\section{Discussion}

Our study showed that though diabetic patients were associated with more co-morbidities and more complex coronary lesions, the CTO revascularization successful rate was similar comparing to that of non-diabetic population. Moreover, successful CTO recanalization was independently associated with reduced risks of allcause mortality rate and adverse cardiovascular events only in diabetic patients, but not in non-diabetic population. These results remained similar in propensity score matching analysis.
Most studies showed that successful recanalization of CTO reduced long term mortality compared to failed procedure or medical therapy and had comparable clinical results to those receiving bypass surgery [13-15]. But, some studies showed conflicting results $[4,16]$. Decision CTO trial reported that there was no difference in long term outcome of successful CTO PCI and optimal medical therapy [4]. However, in this trial, there was high rate of crossover of medical therapy to CTO PCI. Moreover, mean age of patient population was relatively younger (62 years) and proportion of patients with diabetes mellitus (32\%) was lower compared to that of previously reported observational studies [14].

Sanguineti et al. found that diabetes mellitus was a significant predictor of cardiac mortality in patients with CTO lesion. CTO recanalisation reduced major adverse cardiovascular event and suggested a greater reduction in cardiac death among diabetic patients [17]. Failure to recanalize CTO lesion in diabetes was found to have higher residual platelet reactivity (HRPR) which may in turn increase cardiac mortality [18]. In the present study, we found similar result that diabetes mellitus was related to poor prognosis in patients with CTO lesions 


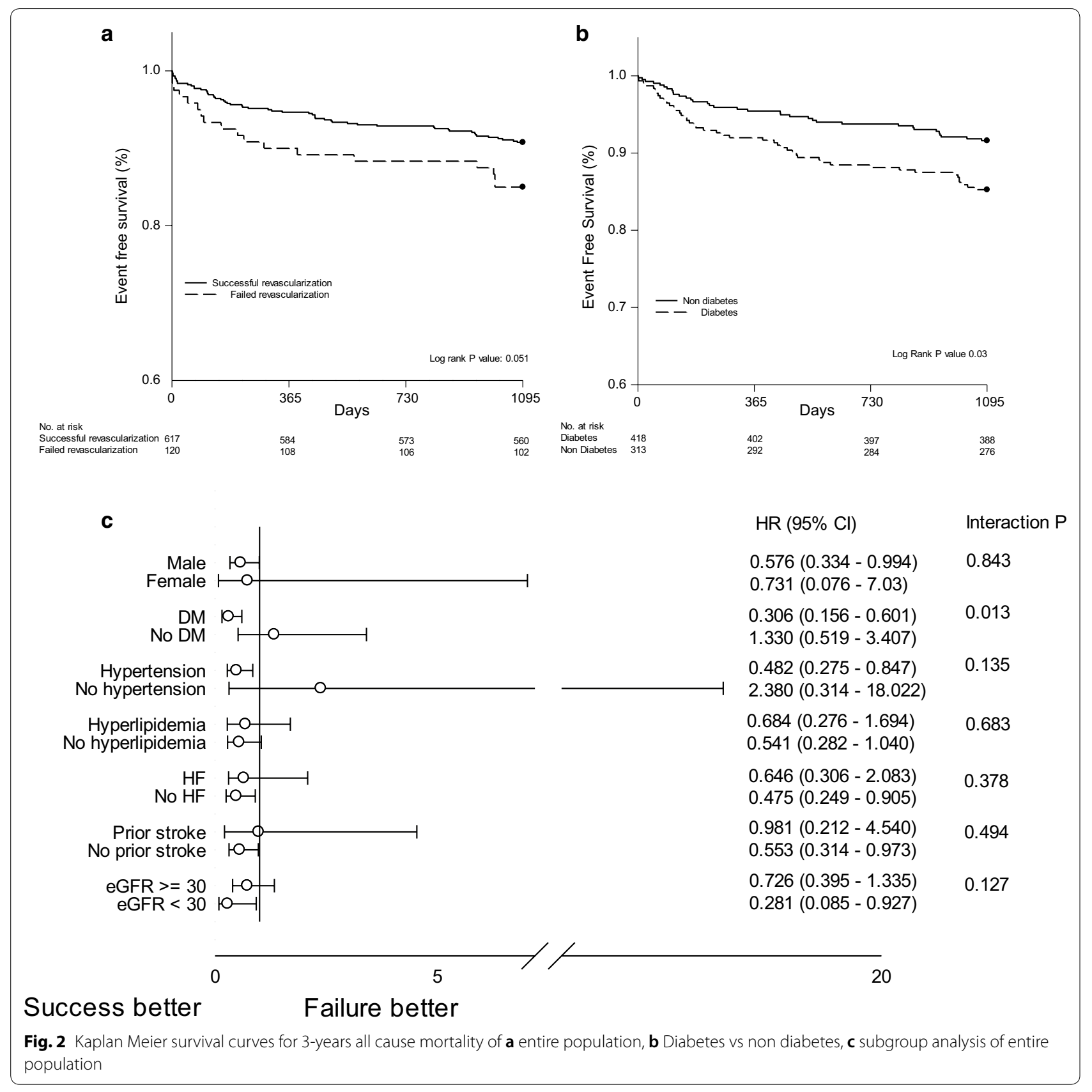

compared to that of non-diabetics. Successful revascularization of CTO lesions in diabetes patients reduced all-cause mortality. But survival benefit was not found in non-diabetics.

Type 2 diabetes mellitus alters glucose and lipid metabolism, leading to premature development of atherosclerosis and adverse outcomes. Prompt recruitment for collateral circulation is crucial to reduce myocardial damage after coronary artery occlusion. Sen et al. found out that diabetes was related to higher incidence of inadequate collateral development in acute coronary syndrome [19]. Potential mechanisms for collateral developments were arteriogenesis, (i.e., arterialization of capillary bed) and angiogenesis [20]. However, chronic hyperglycemia induced microvascular rarefaction in myocardium. It also increases and accumulates advanced glycation endproducts which in turn have negative impact on endothelial function and angiogenesis [21, 22]. Dyslipidemia which is frequently associated with diabetes mellitus confers further greater risk for coronary 
Table 3 Baseline demographics and angiographic characteristics of diabetes and non diabetes population with successful and failed revascularization

\begin{tabular}{|c|c|c|c|c|c|c|}
\hline & \multicolumn{3}{|c|}{ Diabetes mellitus $(n=313)$} & \multicolumn{3}{|c|}{ Non diabetes $(n=426)$} \\
\hline & Success $(n=265)$ & Failed $(n=48)$ & P value & Success $(n=354)$ & Failed $(n=72)$ & $P$ value \\
\hline Age (years) & $70 \pm 12$ & $70 \pm 13$ & 0.71 & $66 \pm 14$ & $68 \pm 13$ & 0.10 \\
\hline Gender (male) & $229(86)$ & $42(88)$ & 1 & $339(96)$ & $65(90)$ & 0.08 \\
\hline Hypertension & $226(85)$ & $42(88)$ & 0.83 & $241(68)$ & $54(75)$ & 0.27 \\
\hline Hyperlipidemia & $121(46)$ & $26(54)$ & 0.35 & $146(41)$ & $27(38)$ & 0.60 \\
\hline Prior stroke & $25(9)$ & $8(17)$ & 0.13 & $26(7)$ & $3(4)$ & 0.45 \\
\hline PAD & $18(7)$ & $7(15)$ & 0.08 & $16(5)$ & $4(6)$ & 0.76 \\
\hline Smoking & $92(35)$ & $17(35)$ & 1.00 & $161(46)$ & $30(42)$ & 0.60 \\
\hline Prior Ml & $77(29)$ & $15(31)$ & 0.73 & $58(16)$ & $9(13)$ & 0.48 \\
\hline Prior PCl & $151(57)$ & $24(50)$ & 0.43 & $166(47)$ & $42(58)$ & 0.09 \\
\hline Prior CABG & $28(11)$ & $8(17)$ & 0.22 & $33(9)$ & $7(10)$ & 0.83 \\
\hline LVEF (\%) & $47 \pm 12$ & $47 \pm 12$ & 0.92 & $50 \pm 12$ & $48 \pm 14$ & 0.36 \\
\hline eGFR, MDRDc (ml/min) & $78 \pm 36$ & $67 \pm 32$ & 0.06 & $89 \pm 29$ & $82 \pm 34$ & 0.06 \\
\hline LDL (mg/dl) & $98 \pm 33$ & $92 \pm 30$ & 0.39 & $106 \pm 36$ & $100 \pm 34$ & 0.29 \\
\hline $\mathrm{HbA1C}$ & $7.9 \pm 1.7$ & $7.7 \pm 1.7$ & 0.57 & - & - & - \\
\hline Non invasive test & & & 0.616 & & & 0.068 \\
\hline Treadmill & $50(19)$ & $6(13)$ & & $80(23)$ & $7(10)$ & \\
\hline Thallium scan & $202(77)$ & $40(83)$ & & $247(70)$ & $61(85)$ & \\
\hline Others & $12(5)$ & $2(4)$ & & $27(7)$ & $4(5)$ & \\
\hline OAD & $191(72)$ & $32(67)$ & 0.62 & - & - & - \\
\hline Metformin & $39(15)$ & $6(13)$ & & & & \\
\hline Sulphonylurea & $15(6)$ & $5(11)$ & & & & \\
\hline DPP4 inhibitor & $5(2)$ & $5(11)$ & & & & \\
\hline Meglitinide & $1(1)$ & $1(2)$ & & & & \\
\hline Combined regimen & $81(31)$ & $12(25)$ & & & & \\
\hline Combined insulin and OAD & $31(12)$ & $4(8)$ & & & & \\
\hline Insulin (\%) & $55(21)$ & $13(27)$ & 0.62 & - & - & - \\
\hline \multicolumn{7}{|l|}{ Location of CTO } \\
\hline LAD & $107(40)$ & $16(33)$ & 0.42 & $164(46)$ & $30(42)$ & 0.52 \\
\hline LCX & $68(26)$ & $16(33)$, & 0.29 & $91(26)$ & $21(29)$ & 0.56 \\
\hline RCA & $138(52)$ & $30(63)$ & 0.21 & $155(44)$ & $33(46)$ & 0.80 \\
\hline J CTO score & $2.54 \pm 1.00$ & $2.66 \pm 1.00$ & 0.38 & $2.31 \pm 0.99$ & $2.40 \pm 0.89$ & 0.537 \\
\hline Syntax score & $18 \pm 6$ & $20 \pm 7$ & 0.03 & $19 \pm 6$ & $20 \pm 7$ & 0.24 \\
\hline MVD & $224(85)$ & $46(96)$ & 0.04 & $277(78)$ & $60(83)$ & 0.43 \\
\hline Lesion length (mm) & $38 \pm 18$ & $31 \pm 9$ & 0.12 & $38+18$ & $32+12$ & 0.05 \\
\hline Lesion width (mm) & $3.0 \pm 1.6$ & $3.0 \pm 0.6$ & 0.96 & $3.3+4.5$ & $3.0+0.3$ & 0.72 \\
\hline Primary retrograde & $22(9)$ & $2(5)$ & 0.85 & $26(8)$ & $6(8)$ & 1.00 \\
\hline
\end{tabular}

Values are given as mean and standard deviation or numbers and percentages

$P C l$ percutaneous coronary intervention, $P A D$ peripheral arterial disease, $M I$ myocardial infarct, $P C l$ percutaneous coronary intervention, $C A B G$ coronary artery bypass graft surgery, $L V E F$ left ventricular ejection fraction, eGFR estimated glomerular filtration rate, $M D R D c$ modification of diet in renal disease Chinese, $L D L$ low density lipoprotein, $H b A 1 C$ glycated hemoglobin, OAD oral anti-diabetic drug, $C T O$ chronic total occlusion, CAD coronary artery disease, $L A D$ left anterior descending, $L C x$ left circumflex, $R C A$ right coronary artery, MVD multivessel significant coronary artery disease

collateralization [23]. Moreover, diabetes mellitus was associated with diffuse atherosclerosis of donor coronary arteries and further impaired collateral circulation over time [24]. These may be possible reasons behind the benefit after successful revascularization in diabetics.
In our study, diabetes patients were older (mean age was 70 years old) and there was high prevalence of chronic kidney disease and heart failure with reduced ejection fraction. Samy et al. found that left ventricular ejection fraction improvement after successful PCI 


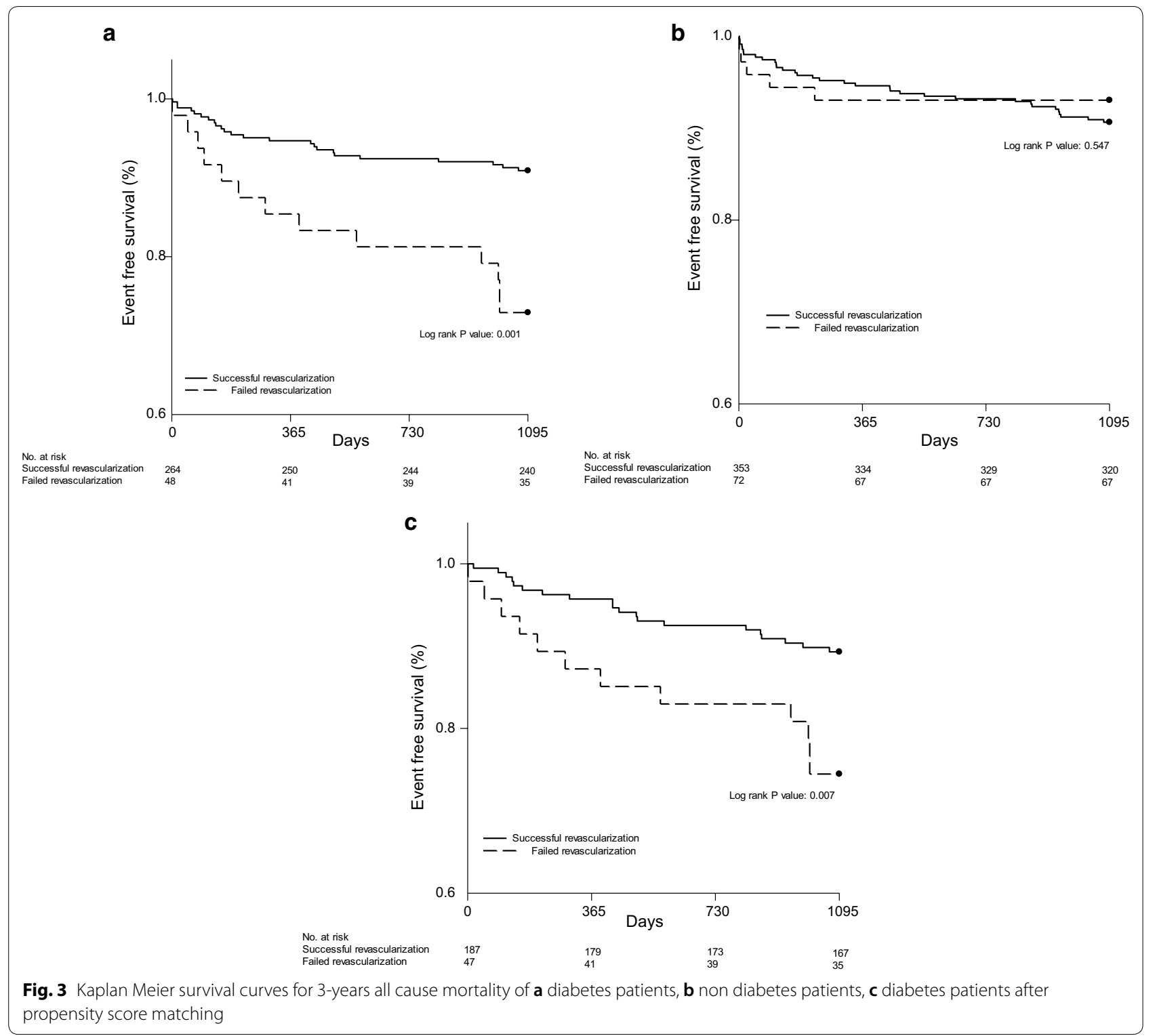

Table 4 Periprocedural complications

\begin{tabular}{|c|c|c|c|c|c|c|}
\hline & \multicolumn{3}{|c|}{ Diabetes patients } & \multicolumn{3}{|c|}{ Non-diabetes patients } \\
\hline & $\begin{array}{l}\text { Successful PCI } \\
(n=265)\end{array}$ & $\begin{array}{l}\text { Failed PCI } \\
(n=48)\end{array}$ & $\overline{P \text { value }}$ & $\begin{array}{l}\text { Successful PCI } \\
(n=354)\end{array}$ & $\begin{array}{l}\text { Failed PCI } \\
(n=72)\end{array}$ & $P$ value \\
\hline In hospital mortality & $1(1)$ & $1(2)$ & 0.28 & $3(1)$ & $2(3)$ & 0.07 \\
\hline Pericardial effusion required pericardiocentesis & $2(1)$ & $0(0)$ & 0.72 & $2(1)$ & $3(4)$ & 0.04 \\
\hline Cardiogenic shock required mechanical support & $2(1)$ & $0(0)$ & 0.72 & $3(1)$ & $1(1)$ & 0.53 \\
\hline CIN required renal replacement therapy & $2(1)$ & $0(0)$ & 0.72 & $1(1)$ & $0(0)$ & 0.83 \\
\hline Vascular complications required surgery & $3(1)$ & $1(2)$ & 0.49 & $3(1)$ & $0(0)$ & 0.57 \\
\hline Periprocedural Ml & $5(2)$ & $3(6)$ & 0.11 & $6(2)$ & $0(0)$ & 0.33 \\
\hline
\end{tabular}

MI myocardial infarct, CIN contrast induced nephropathy 
Table 5 Baseline demographics and angiographic characteristics of diabetes population with successful and failed $\mathrm{PCl}$ after propensity score matching

\begin{tabular}{|c|c|c|c|}
\hline & Success $(n=188)$ & Failed $(n=47)$ & $P$ value \\
\hline Age, years & $71 \pm 12$ & $71 \pm 13$ & 0.96 \\
\hline Gender (Male) & $158(84)$ & $41(87)$ & 0.66 \\
\hline Hypertension & $160(85)$ & $42(89)$ & 0.64 \\
\hline Hyperlipidemia & $80(43)$ & $24(51)$ & 0.33 \\
\hline Prior stroke & $19(10)$ & $6(13)$ & 0.60 \\
\hline PAD & $16(9)$ & $7(15)$ & 0.27 \\
\hline Smoking & $61(32)$ & $17(36)$ & 0.73 \\
\hline Prior Ml & $56(30)$ & $16(34)$ & 0.60 \\
\hline Prior CAD & $111(59)$ & $24(51)$ & 0.33 \\
\hline Prior CABG & $18(10)$ & $9(19)$ & 0.08 \\
\hline LVEF, \% & $47 \pm 12$ & $47 \pm 12$ & 0.92 \\
\hline eGFR, MDRDc (ml/min) & $69 \pm 29$ & $68 \pm 30$ & 0.76 \\
\hline $\mathrm{LDL}(\mathrm{mg} / \mathrm{dl})$ & $98 \pm 34$ & $93 \pm 29$ & 0.51 \\
\hline $\mathrm{HbA} 1 \mathrm{C}, \%$ & $7.7 \pm 1.6$ & $8 \pm 1.5$ & 0.49 \\
\hline \multicolumn{4}{|l|}{ Location of CTO } \\
\hline LAD & $14(26)$ & $5(39)$ & 0.50 \\
\hline LCX & $28(44)$ & $1(11)$ & 0.08 \\
\hline RCA & $23(37)$ & $10(56)$ & 0.18 \\
\hline Multivessel CTO & $29(15)$ & $15(32)$ & 0.05 \\
\hline J CTO score & $2.4 \pm 0.5$ & $2.5 \pm 0.6$ & 0.60 \\
\hline Syntax score & $18 \pm 5$ & $19 \pm 5$ & 0.78 \\
\hline MVD & $163(87)$ & $45(96)$ & 0.05 \\
\hline Lesion length (mm) & $37 \pm 18$ & $31 \pm 8$ & 0.12 \\
\hline Lesion width (mm) & $3.0 \pm 2.0$ & $3.0 \pm 0.6$ & 0.92 \\
\hline Retrograde approach & $14(8)$ & $2(5)$ & 0.89 \\
\hline
\end{tabular}

Values are given as mean and standard deviation or numbers and percentages $P C l$ percutaneous coronary intervention, $P A D$ peripheral arterial disease, $C A B G$ coronary artery bypass graft surgery, LVEF left ventricular ejection fraction, eGFR estimated glomerular filtration rate, $L D L$ low density lipoprotein, CTO chronic total occlusion, $C A D$ coronary artery disease, $L A D$ left anterior descending, $L C X$ left circumflex, $R C A$ right coronary artery, $M V D$ multivessel significant coronary artery disease

was significantly more in patient with lower ejection fraction group [25]. Galassi et al. also reported that successful revascularization in patients with left ventricular ejection fraction $\leq 35 \%$ improved left ventricular ejection fraction and 2 years all-cause mortality [26]. Moreover, successful PCI was associated with better cardiac survival in elderly especially when complete revascularization is achieved [27]. Recently, Yunfeng et al. also reported that successful revascularization of CTO of stable right coronary artery either by PCI or bypass graft showed significant reduction of all-cause mortality (HR: 0.429, 95\% CI 0.269-0.682) [28]. These evidences highlighted the importance of complete revascularization. Benefit of recanalization of CTO may be more pronounced in patients with elderly and poor left ventricular ejection fraction. Chronic kidney disease was one of poor prognostic factors for patients with CTO [29]. Diabetes mellitus is one of well-known underlying diseases that lead to chronic kidney disease. However, successful CTO PCI was associated with better survival irrespective of renal function status of patient [30].

Coronary revascularization of CTO lesion is always complex and demands delicate techniques, ample experiences and familiarity to special devices. Moreover, it is associated with higher perioperative complications such as coronary artery perforation, contrast induced nephropathy, radiation hazard, and mortality. Diabetes mellitus is associated with multi-, small vessel, diffuse atherosclerotic disease and higher rate of periprocedural MI, contrast induced nephropathy which may impact on procedure success rate and complications that consequently affect long term outcomes. However, in Bypass Angioplasty Revascularization Investigation 2 Diabetes trial, CTO didn't increase periprocedural mortality in diabetes patients treated either by PCI or bypass surgery but it was associated with poor prognosis if left untreated [31, 32]. In our study, technical success rate of revascularization of CTO in diabetics was not different as compare to that of non-diabetics with similar peri-procedural complications. OPEN CTO trial was a prospective multi center registry evaluating about procedural success rate and complications [33]. This trial had also shown successful revascularization rate of $86 \%$ and reported no difference in technical outcomes between diabetics and non-diabetics. Taken together, these evidences suggest that CTO in diabetes patients should not preclude the CTO PCI attempt.

\section{Limitations}

Our study had some limitations. First, all patients in our study received coronary revascularization for CTO. There was no control group that received optimal medical therapy or coronary artery bypass graft to compare outcome. Second, it is a retrospective, nonrandomized and observational study. Although we performed propensity score matching to reduce potential bias, the result cannot be comparable to that of randomized trial. Third, our study was conducted in a tertiary medical center that performed high volume of percutaneous CTO revascularization. Our result may not be applicable in low volume and less experienced center. Fourth, due to high proportion (91\%) of male gender in our study, it's application on female gender may be limited. Fifth, as some of our patients were referred to local hospital after intervention, some of follow up information may not be available when our research coordinator couldn't reach them. Sixth, bare metallic stents were implanted 
Table 6 Univariate and multivariate analysis of successful revascularization on 3-years all cause mortality before matching

\begin{tabular}{|c|c|c|c|c|}
\hline & \multicolumn{2}{|l|}{ Univariate analysis } & \multicolumn{2}{|l|}{ Multivariate analysis } \\
\hline & $\mathrm{HR}(95 \% \mathrm{Cl})$ & P value & $\mathrm{HR}(95 \% \mathrm{Cl})$ & $P$ value \\
\hline Successful revascularization & $0.307(0.156-0.604)$ & 0.001 & $0.289(0.125-0.667)$ & 0.004 \\
\hline Age & $1.044(1.015-1.075)$ & 0.003 & $1.034(0.997-1.073)$ & 0.071 \\
\hline Gender & $3.607(0.873-14.902)$ & 0.076 & - & - \\
\hline eGFR & $0.990(0.981-1.000)$ & 0.053 & $0.996(0.985-1.007)$ & 0.465 \\
\hline LDL & $0.991(0.979-1.003)$ & 0.150 & - & - \\
\hline $\mathrm{HbA1C}$ & $0.830(0.637-1.081)$ & 0.166 & - & - \\
\hline Prior stroke & $2.409(1.158-5.012)$ & 0.019 & $1.961(0.725-5.308)$ & 0.185 \\
\hline Prior PAD & 3.109 (1.494-6.469) & 0.002 & $3.322(1.276-8.646)$ & 0.014 \\
\hline Prior Ml & $1.147(0.576-2.282)$ & 0.697 & & \\
\hline Hypertension & $1.302(0.513-3.302)$ & 0.579 & - & - \\
\hline Multivessel disease & $1.205(0.746-1.946)$ & 0.445 & & \\
\hline LAD & $1.575(0.872-2.846)$ & 0.132 & - & - \\
\hline Syntax score & $1.054(0.995-1.117)$ & 0.074 & & \\
\hline LVEF & $0.960(0.933-0.988)$ & 0.005 & $0.955(0.927-0.984)$ & 0.003 \\
\hline
\end{tabular}

MDRDC Modification of diet in renal disease Chinese, $L D L$ low density lipoprotein, $H b A 1 C$ glycated hemoglobulin, $P A D$ peripheral arterial disease, $L A D$ left anterior descending, LVEF left ventricular ejection fraction

Table 7 Univariate and multivariate analysis of successful revascularization on 3-years all cause mortality after matching

\begin{tabular}{|c|c|c|c|c|}
\hline & \multicolumn{2}{|l|}{ Univariate analysis } & \multicolumn{2}{|l|}{ Multivariate analysis } \\
\hline & $\mathrm{HR}(95 \% \mathrm{Cl})$ & $P$ value & $\mathrm{HR}(95 \% \mathrm{Cl})$ & $P$ value \\
\hline Successful revascularization & $0.386(0.188-0.789)$ & 0.009 & $0.348(0.142-0.851)$ & 0.021 \\
\hline Age & $1.045(1.010-1.081)$ & 0.012 & $1.041(0.997-1.087)$ & 0.069 \\
\hline Gender & $2.789(0.667-11.673)$ & 0.160 & - & - \\
\hline eGFR & 0.987 (0.975-0.999) & 0.040 & $0.995(0.979-1.011)$ & 0.516 \\
\hline LDL & $0.993(0.980-1.007)$ & 0.346 & - & - \\
\hline $\mathrm{HbA1C}$ & $0.671(0.386-1.169)$ & 0.159 & - & - \\
\hline Prior stroke & $2.598(1.123-6.009)$ & 0.026 & $2.207(0.736-6.621)$ & 0.158 \\
\hline Prior PAD & $1.825(0.703-4.740)$ & 0.217 & - & - \\
\hline Prior Ml & $1.176(0.567-2.440)$ & 0.662 & & \\
\hline Hypertension & $1.603(0.488-5.261)$ & 0.437 & - & - \\
\hline Multi vessel disease & $0.680(0.226-2.048)$ & 0.493 & & \\
\hline LAD & $0.194(0.024-1.577)$ & 0.125 & - & - \\
\hline Syntax score & $1.024(0.888-1.180)$ & 0.745 & & \\
\hline LVEF & 0.965 (0.933-0.999) & 0.045 & $0.961(0.928-0.996)$ & 0.027 \\
\hline
\end{tabular}

$M D R D c$ Modification of diet in renal disease Chinese, $L D L$ low density lipoprotein, $H b A 1 C$ glycated hemoglobulin, $P A D$ peripheral arterial disease, $L A D$ left anterior descending, $L V E F$ left ventricular ejection fraction

in some patients due to personal economic issue or contraindication to prolonged dual antiplatelet therapy. Next generation drug eluting stent and recent trial about short term dual antiplatelet therapy may solve this problem in future. Seventh, patients with non-diabetics were younger and lesser co-morbidities compared to that of diabetics so that longer follow up period may be necessary to find potential benefit from successful CTO PCI to avoid potential type 2 error. Moreover, data about contrast volume and fluoroscopy time is missing.

\section{Conclusions}

The CTO revascularization successful rate was similar between diabetic and non-diabetic population. Successful CTO recanalization was found to be associated with improved clinical outcomes in diabetic patients. 
However, the benefit of CTO PCI didn't outweigh the risk of failed procedure in non-diabetics. Further randomized controlled trial and longer term follow up are necessary to confirm our results.

\begin{abstract}
Abbreviations
CTO: Chronic total occlusion; PCI: Percutaneous coronary intervention; MACE: Major adverse cardiovascular events; TIMI: Thrombolysis in myocardial infarction; DM: Diabetes mellitus; eGFR: Estimated glomerular filtration rate; MDRDc: Modified diet in renal disease equation for Chinese; CART: Controlled antegrade and retrograde subintimal tracking; CV: Cardiovascular; SD: Standard deviation; LVEF: Left ventricular ejection fraction.
\end{abstract}

\section{Acknowledgements}

Not applicable.

\section{Authors' contributions}

Dataset was collected by TCT and HWJ. Data was interpreted and analyzed by TCT and HWJ with help from LTM. Manuscript was drafted by TCT and LTM. All authors read and approved the final manuscript.

\section{Funding}

Not applicable.

\section{Availability of data and materials}

The dataset used and analyzed during the current study are available from corresponding author on request.

\section{Ethics approval and consent to participate}

This study was approved by the Research Ethics Committee of Taipei Veterans General Hospital. Written informed consent was obtained from all patients or their legal representatives.

\section{Consent for publication}

No individual participant data were reported that would require consent from the participant to publish.

\section{Competing interests}

The authors declare that they have no competing interests.

\section{Author details}

1 Division of Cardiology, Department of Medicine, Taipei Veterans General Hospital, No. 201, Sec. 2, Shih-Pai Road, Taipei, Taiwan. ${ }^{2}$ Cardiovascular Research Center, National Yang-Ming University, Taipei, Taiwan. ${ }^{3}$ Institute of Clinical Medicine, National Yang-Ming University, Taipei, Taiwan.

Received: 15 May 2020 Accepted: 25 July 2020

Published online: 01 August 2020

\section{References}

1. Christofferson RD, Lehmann KG, Martin GV, Every N, Caldwell JH, Kapadia SR. Effect of chronic total coronary occlusion on treatment strategy. Am J Cardiol. 2005;95(9):1088-91.

2. Fefer P, Knudtson ML, Cheema AN, Galbraith PD, Osherov AB, Yalonetsky S, Gannot S, Samuel M, Weisbrod M, Bierstone D, et al. Current perspectives on coronary chronic total occlusions: the Canadian Multicenter Chronic Total Occlusions Registry. J Am Coll Cardiol. 2012;59(11):991-7.

3. Ma Y, Li D, Li J, Li Y, Bai F, Qin F, Zhou S, Liu Q. Percutaneous coronary intervention versus optimal medical therapy for patients with chronic total occlusion: a meta-analysis and systematic review. J Thorac Dis. 2018;10(5):2960-7.

4. Lee SW, Lee PH, Ahn JM, Park DW, Yun SC, Han S, Kang H, Kang SJ, Kim $\mathrm{YH}$, Lee CW, et al. Randomized trial evaluating percutaneous coronary intervention for the treatment of chronic total occlusion. Circulation. 2019;139(14):1674-83.
5. Roffi M, Angiolillo DJ, Kappetein AP. Current concepts on coronary revascularization in diabetic patients. Eur Heart J. 2011;32(22):2748-57.

6. Roffi M, Iglesias JF. CTO PCl in patients with diabetes mellitus: sweet perspectives. JACC Cardiovasc Interv. 2017;10(21):2182-4.

7. Salisbury AC, Sapontis J, Grantham JA, Qintar M, Gosch KL, Lombardi W, Karmpaliotis D, Moses J, Cohen DJ, Spertus JA, et al. Outcomes of chronic total occlusion percutaneous coronary intervention in patients with diabetes: insights from the OPEN CTO Registry. JACC Cardiovasc Interv. 2017;10(21):2174-81.

8. Sohrabi B, Ghaffari S, Habibzadeh A, Chaichi P. Outcome of diabetic and non-diabetic patients undergoing successful percutaneous coronary intervention of chronic total occlusion. J Cardiovasc Thorac Res. 2011:3(2):45-8.

9. Mashaly A, Rha SW, Choi BG, Baek MJ, Ryu YG, Choi SY, Byun JK, Li H, Shim MS, Jang WY, et al. Impact of diabetes mellitus on 5-year clinical outcomes in patients with chronic total occlusion lesions. Coron Artery Dis. 2018;29(2):119-26.

10. Erratum. Classification and diagnosis of diabetes. Sec. 2. In standards of medical care in diabetes-2016. Diabetes Care. 2016;39(Suppl. 1):S13-S22. Diabetes Care. 2016;39(9):1653.

11. Kuo CF, Yu KH, Shen YM, See LC. The Chinese version of the modification of diet in renal disease (MDRD) equation is a superior screening tool for chronic kidney disease among middle-aged Taiwanese than the original MDRD and Cockcroft-Gault equations. Biomed J. 2014;37(6):398-405.

12. Christopoulos G, Wyman RM, Alaswad K, Karmpaliotis D, Lombardi W, Grantham JA, Yeh RW, Jaffer FA, Cipher DJ, Rangan BV, et al. Clinical utility of the Japan-chronic total occlusion score in coronary chronic total occlusion interventions: results from a multicenter registry. Circ Cardiovasc Interv. 2015;8(7):e002171.

13. Kim BS, Yang JH, Jang WJ, Song YB, Hahn JY, Choi JH, Kim WS, Lee YT, Gwon HC, Lee SH, et al. Clinical outcomes of multiple chronic total occlusions in coronary arteries according to three therapeutic strategies: bypass surgery, percutaneous intervention and medication. Int J Cardiol. 2015;197:2-7.

14. Tsai TT, Stanislawski MA, Shunk KA, Armstrong EJ, Grunwald GK, Schob AH, Valle JA, Alfonso CE, Nallamothu BK, Ho PM, et al. Contemporary incidence, management, and long-term outcomes of percutaneous coronary interventions for chronic coronary artery total occlusions: insights from the VA CART program. JACC Cardiovasc Interv. 2017;10(9):866-75.

15. Roth C, Goliasch G, Aschauer S, Gangl C, Ayoub M, Distelmaier K, Frey B, Lang IM, Berger R, Mashayekhi K, et al. Impact of treatment strategies on long-term outcome of CTO patients. Eur J Intern Med. 2020;77:97-104.

16. Lee PH, Lee SW, Park HS, Kang SH, Bae BJ, Chang M, Roh JH, Yoon SH, Ahn $J M$, Park DW, et al. Successful recanalization of native coronary chronic total occlusion is not associated with improved long-term survival. JACC Cardiovasc Interv. 2016;9(6):530-8.

17. Sanguineti F, Garot P, O'Connor S, Watanabe Y, Spaziano M, Lefevre T, Hovasse T, Benamer H, Unterseeh T, Chevalier B, et al. Chronic total coronary occlusion treated by percutaneous coronary intervention: longterm outcome in patients with and without diabetes. Eurolntervention. 2017;12(15):e1889-97.

18. Valenti R, Cantini G, Marcucci R, Marrani M, Migliorini A, Carrabba N, Comito V, Vergara R, Cerisano G, Parodi G, et al. Prognostic impact of high residual platelet reactivity after chronic total occlusion percutaneous coronary intervention in patients with diabetes mellitus. Int J Cardiol. 2015;201:561-7.

19. Şen Ö, Allahverdiyev S, Topuz M, Baykan AO, Oz F, Koç M. Clinical significance and determinants of prompt recruitment collaterals during primary percutaneous coronary intervention. Kardiol Pol. 2017;75(8):763-9.

20. Carmeliet P. Mechanisms of angiogenesis and arteriogenesis. Nat Med. 2000;6(4):389-95.

21. Shen Y, Ding FH, Dai Y, Wang XQ, Zhang RY, Lu L, Shen WF. Reduced coronary collateralization in type 2 diabetic patients with chronic total occlusion. Cardiovasc Diabetol. 2018;17(1):26.

22. Du R, Zhang RY, Lu L, Shen Y, Pu LJ, Zhu ZB, Zhang Q, Hu J, Yang ZK, Ding $\mathrm{FH}$, et al. Increased glycated albumin and decreased esRAGE levels in serum are related to negative coronary artery remodeling in patients with type 2 diabetes: an Intravascular ultrasound study. Cardiovasc Diabetol. 2018;17(1):149.

23. Shen $Y$, Chen $S$, Dai $Y$, Wang $X Q$, Zhang RY, Yang ZK, Hu J, Lu L, Ding FH, Shen WF. Lipoprotein (a) interactions with cholesterol-containing lipids 
on angiographic coronary collateralization in type 2 diabetic patients with chronic total occlusion. Cardiovasc Diabetol. 2019;18(1):82.

24. Shen Y, Yang ZK, Hu J, Wang XQ, Dai Y, Zhang S, Zhang RY, Lu L, Ding FH, Shen WF. Donor artery stenosis interactions with diastolic blood pressure on coronary collateral flow in type 2 diabetic patients with chronic total occlusion. Cardiovasc Diabetol. 2018;17(1):76.

25. Samy M, El Awady WS, Al-Daydamony MM, Abd El Samei MM, Shokry K. Echocardiographic assessment of left ventricular function recovery post percutaneous coronary intervention of chronic total occlusions in patients with low and mid-range left ventricular ejection fractions. Echocardiography. 2020;37:239-46.

26. Galassi AR, Boukhris M, Toma A, Elhadj Z, Laroussi L, Gaemperli O, Behnes M, Akin I, Luscher TF, Neumann FJ, et al. Percutaneous coronary intervention of chronic total occlusions in patients with low left ventricular ejection fraction. JACC Cardiovasc Interv. 2017;10(21):2158-70.

27. Valenti R, Migliorini A, De Gregorio MG, Martone R, Berteotti M, Bernardini A, Carrabba N, Vergara R, Marchionni N, Antoniucci D. Impact of complete percutaneous revascularization in elderly patients with chronic total occlusion. Catheter Cardiovasc Interv. 2020;95(1):145-53.

28. Yan Y, Zhang M, Yuan F, Liu H, Wu D, Fan Y, Guo X, Xu F, Zhang M, Zhao $Q$, et al. Successful revascularization versus medical therapy in diabetic patients with stable right coronary artery chronic total occlusion: a retrospective cohort study. Cardiovasc Diabetol. 2019;18(1):108.

29. Mehran R, Claessen BE, Godino C, Dangas GD, Obunai K, Kanwal S, Carlino M, Henriques JP, Di Mario C, Kim YH, et al. Long-term outcome of percutaneous coronary intervention for chronic total occlusions. JACC Cardiovasc Interv. 2011;4(9):952-61.
30. Malik AO, Spertus JA, Grantham JA, Peri-Okonny P, Gosch K, Sapontis J, Moses J, Lombardi W, Karmpaliotis D, Nicholson WJ, et al. Outcomes of chronic total occlusion percutaneous coronary intervention in patients with renal dysfunction. Am J Cardiol. 2020;125(7):1046-53.

31. Damluji AA, Pomenti SF, Ramireddy A, Al-Damluji MS, Alfonso CE, Schob AH, Marso SP, Gilchrist IC, Moscucci M, Kandzari DE, et al. Influence of total coronary occlusion on clinical outcomes (from the Bypass Angioplasty Revascularization Investigation 2 DiabetesTrial). Am J Cardiol. 2016;117(7):1031-8

32. Tajti P, Karmpaliotis D, Alaswad K, Jaffer FA, Yeh RW, Patel M, Mahmud E, Choi JW, Burke MN, Doing AH, et al. In-hospital outcomes of chronic total occlusion percutaneous coronary interventions in patients with prior coronary artery bypass graft surgery. Circ Cardiovasc Interv. 2019;12(3):e007338

33. Sapontis J, Salisbury AC, Yeh RW, Cohen DJ, Hirai T, Lombardi W, McCabe JM, Karmpaliotis D, Moses J, Nicholson WJ, et al. Early procedural and health status outcomes after chronic total occlusion angioplasty: a report from the OPEN-CTO Registry (Outcomes, Patient Health Status, and Efficiency in Chronic Total Occlusion Hybrid Procedures). JACC Cardiovasc Interv. 2017;10(15):1523-34

\section{Publisher's Note}

Springer Nature remains neutral with regard to jurisdictional claims in published maps and institutional affiliations.
Ready to submit your research? Choose BMC and benefit from:

- fast, convenient online submission

- thorough peer review by experienced researchers in your field

- rapid publication on acceptance

- support for research data, including large and complex data types

- gold Open Access which fosters wider collaboration and increased citations

- maximum visibility for your research: over $100 \mathrm{M}$ website views per year

At BMC, research is always in progress.

Learn more biomedcentral.com/submissions 